\title{
Comprehensive modeling of the band gap and absorption spectrum of $\mathrm{BiVO}_{4}$
}

\author{
Julia Wiktor, ${ }^{*}$ Igor Reshetnyak, Francesco Ambrosio, and Alfredo Pasquarello \\ Chaire de Simulation à l'Echelle Atomique (CSEA), Ecole Polytechnique Fédérale de Lausanne (EPFL), \\ CH-1015 Lausanne, Switzerland
}

(Received 2 May 2017; published 12 July 2017)

\begin{abstract}
We present first-principles calculations of the electronic structure of $\mathrm{BiVO}_{4}$ at various levels of theory. In the calculations, we take into account a series of effects that affect the band gap, i.e., spin-orbit coupling, electron-hole interaction, nuclear quantum motions, and thermal vibrations. All these effects lead to a significant renormalization of the band gap. After including the relevant corrections, the values achieved with the $G W$ level of theory closely match the experiment. Additionally, by treating excitonic effects through the Bethe-Salpeter equation, we obtain an optical band gap and an absorption spectrum in good agreement with experimental data. Through the calculation of Tauc plots, we show that this technique gives the optical band gap within about $0.14 \mathrm{eV}$, but argue that it is unable to distinguish between direct and indirect bad gaps in the case of $\mathrm{BiVO}_{4}$.
\end{abstract}

DOI: 10.1103/PhysRevMaterials.1.022401

Several materials among complex metal oxides have shown excellent photoelectrochemical properties and stability in harsh conditions, ideal for application as photoanodes of watersplitting cells $[1,2]$. Among them, monoclinic bismuth vanadate $\left(\mathrm{m}-\mathrm{BiVO}_{4}\right)$ has attracted early attention [3-6]. $\mathrm{BiVO}_{4}$ is a nontoxic and chemically stable material, which has the ability of absorbing a large portion of the visible spectrum, due to its band gap of 2.4-2.5 eV [7-11]. Moreover, $\mathrm{BiVO}_{4}$ has band edges favorably positioned with respect to the water redox potentials [12] and exhibits high photon-to-current conversion efficiencies $[13,14]$. The good photocatalytic activity under visible light of $\mathrm{BiVO}_{4}$ has led to extensive experimental [7-11] and computational [15-19] studies.

There are several density functional theory (DFT) studies on the electronic structure of $\mathrm{BiVO}_{4}$ [2,15-19]. It was generally observed that the results obtained with the semilocal Perdew-Burke-Ernzerhof (PBE) functional [20] or with hybrid functionals with a reduced fraction of nonlocal Fock exchange are in much better agreement with experiments than standard hybrid functionals. This observation is surprising, since semilocal functionals generally underestimate band gaps of semiconductors significantly, while standard hybrid functionals lead to much better agreement with experiment [21]. The understanding of the underlying reasons for this behavior is crucial in view of the ongoing high-throughput efforts for identifying the most efficient photoanode materials among the metal vanadates [2].

In this Communication, we perform calculations at various levels of theory, including hybrid functionals [22,23] and the many-body perturbation theory based on Hedin's $G W$ formulation [24]. We show that the agreement observed in semilocal DFT calculations is artificial, since various effects leading to the reduction of the band gap have not been considered. We find that taking into account spin-orbit coupling (SOC), excitonic effects, nuclear quantum effects (NQEs), and thermal vibrations leads to a band gap reduction of about $1.1 \mathrm{eV}$. Additionally, we carry out a close comparison with the experimental procedure for extracting the band gap of $\mathrm{BiVO}_{4}$. The experimental band gaps of semiconductors are usually extracted from

*julia.wiktor@epfl.ch
UV-vis optical absorption spectroscopy, through the linear extrapolation of Tauc plots [25]. By deriving Tauc plots from the calculated absorption spectrum, we show that the method can lead to slight band-gap overestimation. Additionally, as excitonic effects are found to be significant in $\mathrm{BiVO}_{4}$, we emphasize that Tauc plots cannot be used to distinguish between direct and indirect gaps in the case of this material.

The electronic structure calculations presented here are carried out within various frameworks. We begin with calculations within density functional theory (DFT) based on the semilocal Perdew-Burke-Ernzerhof (PBE) functional. Next, we use two types of hybrid functionals, Perdew-Burke-Ernzerhof (PBE0) [23], and Heyd-Scuseria-Ernzerhof (HSE) [22,26], in which $25 \%$ of PBE exchange is replaced by nonlocal Fock exchange. We also turn to the many-body perturbation theory based on Hedin's $G W$ scheme [24]. We carry out one-shot $G_{0} W_{0}$ calculations, both starting from wave functions obtained at the semilocal and hybrid functional levels. Finally, we apply quasiparticle self-consistent QS $G \tilde{W}$ including an efficient exchange-correlation kernel to account for vertex corrections, as described in Ref. [27]. This method was shown to yield band gaps agreeing with experiment within $0.13 \mathrm{eV}$, on average. Core-valence interactions in all calculations are treated through norm-conserving pseudopotentials [28]. We include 15 valence electrons for $\mathrm{Bi}(5 d, 6 s$, and $6 p), 13$ for $\mathrm{V}(3 s, 3 p, 3 d$, and $4 s)$ and 6 for $\mathrm{O}(2 s$ and $2 p)$. The calculations are performed for the experimental structure at room temperature taken from Ref. [29], with atomic positions relaxed at the PBE level. We consider a unit cell containing 12 atoms to model the monoclinic phase of $\mathrm{BiVO}_{4}$. The structure of $\mathrm{m}-\mathrm{BiVO}_{4}$ is given in Fig. 1. We use a plane-wave cut-off energy of $42 \mathrm{Ha}$ and a k-point mesh of $4 \times 4 \times 4$. These parameters ensure the convergence of the total energy to less than $1 \mathrm{meV}$ per atom. We remark that the regular $\mathbf{k}$-point mesh used here does not include the smallest gap of $\mathrm{BiVO}_{4}$ (see Fig. 2). However, at the PBE level, the direct gap located at the $(0.5,0,0.25)$ point is only $0.13 \mathrm{eV}$ larger than the lowest indirect energy transition. From the QS $G \tilde{W}$ band structure, we find that the difference between the two transitions amounts to $0.16 \mathrm{eV}$. We systematically account for this reduction in the band gaps achieved with hybrid functionals and $G W$ methods. In the $G W$ calculations, we include about 1000 empty bands. 

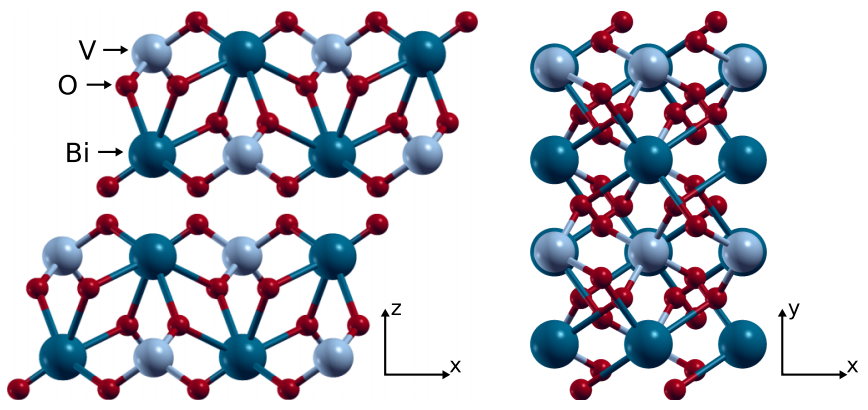

FIG. 1. Representation of the crystal structure of monoclinic $\mathrm{BiVO}_{4}$.

The frequency dependence of the dielectric matrix is evaluated by the contour deformation method [30], through the use of 8 real and 4 imaginary frequencies, and a plane-wave cutoff of $8 \mathrm{Ha}$. We note that the relatively small value of the cut-off needed to describe the dielectric function results from the use of the contour deformation method [31]. We performed convergence tests on the quasiparticle energies by increasing the aforementioned parameters. From these tests we estimate that the $G W$ band gaps are converged within $0.05 \mathrm{eV}$. In the QS $G \tilde{W}$, calculations we update and diagonalize the lowest 100 bands. The calculations for the fundamental band gap are performed with the ABINIT code [32-34].

We first present results for the fundamental band gap, without the effects of spin-orbit coupling, electron-hole interaction, or atomic vibrations. Band gaps yielded by various methods are given in Table I. The value of $2.18 \mathrm{eV}$ achieved with the low-level PBE functional apparently shows best agreement with experimental values (2.4-2.5 eV), as was observed in previous studies [15-19]. The use of hybrid functionals leads to much higher values, especially in the case of PBE0 $(3.92 \mathrm{eV})$. Various calculations performed at the $G W$ level

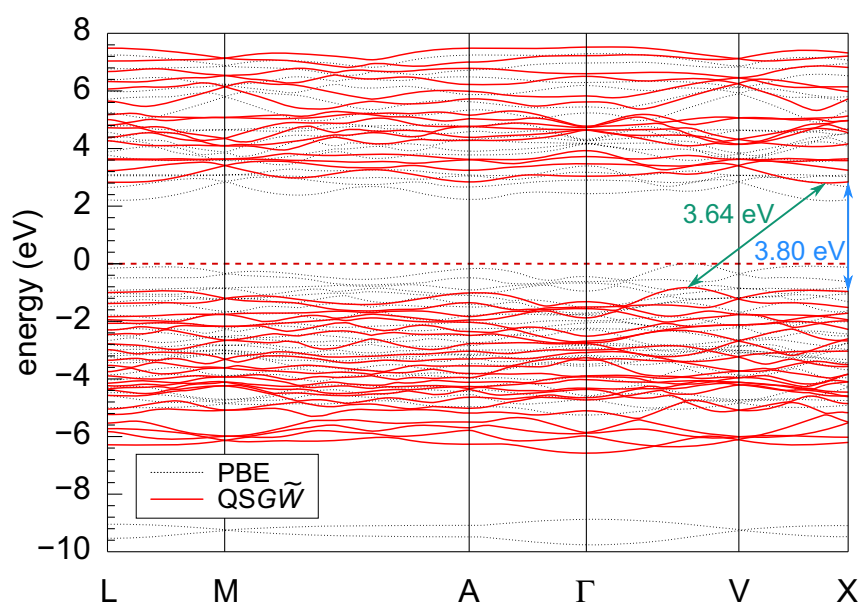

FIG. 2. Band structure of $\mathrm{m}-\mathrm{BiVO}_{4}$ calculated using the PBE functional (black dashed line) and the QSG $\tilde{W}$ method (red solid lines). The QSG $\tilde{W}$ band structure is generated assuming, for each group of bands, a linear relationship between the $G W$ corrections and the energy. The energies are referred to the PBE valence-band maximum. The point $X$ corresponds to $(0.5,0,0.25)$ in reduced units, for the positions of the other points see Ref. [16].
TABLE I. Fundamental and optical band gap of $\mathrm{BiVO}_{4}$ as obtained with various methods. The fundamental gap $E_{\mathrm{g}}$ does not account for the effects of spin-orbit coupling, electron-hole interaction or atomic vibrations. The fundamental gap $E_{\mathrm{g}}^{\text {corr. }}$ includes corrections from spin-orbit coupling, nuclear quantum effects, and thermal vibrations. $E_{\mathrm{g}}^{\text {opt. }}$ corresponds to the optical band gap. $\tilde{W}_{0}$ and $\tilde{W}$ correspond to the inclusion of the vertex corrections in the screening.

\begin{tabular}{lccc}
\hline \hline & $E_{\mathrm{g}}(\mathrm{eV})$ & $E_{\mathrm{g}}^{\text {corr. }}(\mathrm{eV})$ & $E_{\mathrm{g}}^{\text {opt. }}(\mathrm{eV})$ \\
\hline PBE & 2.18 & 1.48 & 1.37 \\
$\mathrm{HSE}$ & 3.18 & 2.13 & 2.02 \\
$\mathrm{PBE} 0$ & 3.92 & 2.87 & 2.76 \\
$G_{0} W_{0} @ \mathrm{PBE}$ & 3.52 & 2.47 & 2.36 \\
$G_{0} W_{0} @ \mathrm{HSE}$ & 3.68 & 2.63 & 2.52 \\
$G_{0} W_{0} @ \mathrm{PBE} 0$ & 3.98 & 2.93 & 2.82 \\
$G_{0} \tilde{W}_{0} @ \mathrm{PBE}$ & 3.39 & 2.34 & 2.23 \\
$G_{0} \tilde{W}_{0} @ \mathrm{HSE}$ & 3.43 & 2.38 & 2.27 \\
$G_{0} \tilde{W}_{0} @ \mathrm{PBE} 0$ & 3.63 & 2.58 & 2.47 \\
$\mathrm{QS} G \tilde{W}$ & 3.64 & 2.59 & 2.48 \\
Expt. & & & $2.4-2.5[7-11]$ \\
\hline \hline
\end{tabular}

predict similar values in the range 3.39-3.98 eV, much higher than the experimental results. However, in these calculations many effects that could affect the electronic structure are not accounted for.

Since bismuth is a heavy element, spin-orbit coupling can significantly affect its electronic structure. To evaluate the effect of SOC on the band gap, we perform fully relativistic calculations with two-component spinor wave functions at the PBE level. We find that the spin-orbit coupling leads to a decrease of the band gap by $0.13 \mathrm{eV}$. This effect is rather small, since the band edges of bismuth vanadate mostly comprise $\mathrm{O}$ $2 p$ and $\mathrm{V} 3 d$ states, which are only indirectly affected by the shift of the bismuth energy levels. In the corrected band gaps at the higher levels of theory, the shift calculated at the PBE level has been included. Considering the nature of the band edges of $\mathrm{BiVO}_{4}(\mathrm{O} 2 p$ and $\mathrm{V} 3 d$ states), we expect such a treatment to be sufficient.

The experimental gaps reported in the literature were measured at room temperature, while the calculations described above are carried out at $0 \mathrm{~K}$, with fixed atomic positions. Atomic vibrations due to finite temperatures can lead to significant renormalization of the band gap [35]. To take this effect into account, we carry out 5 ps long molecular dynamics (MD) simulations with classical nuclei in the canonical $N V T$ ensemble at a temperature of $300 \mathrm{~K}$. We performed these calculations with the $\mathrm{CP} 2 \mathrm{~K}$ code [36]. Atom-centered Gaussian-type basis functions are used to describe the orbitals and an auxiliary plane-wave basis set is employed to re-expand the electron density. We employ the MOLOPT basis set [37] for $\mathrm{Bi}, \mathrm{O}$, and $\mathrm{V}$, and use a cutoff of $600 \mathrm{Ha}$ for the plane waves. Core-valence interactions are described by Goedecker-Teter-Hutter pseudopotentials [38]. In this simulation, we use an orthorhombic supercell containing 192 atoms $(a=10.39, b=10.18$, and $c=23.39 \AA)$ and sample the Brillouin zone at the sole $\Gamma$ point. The time step is 

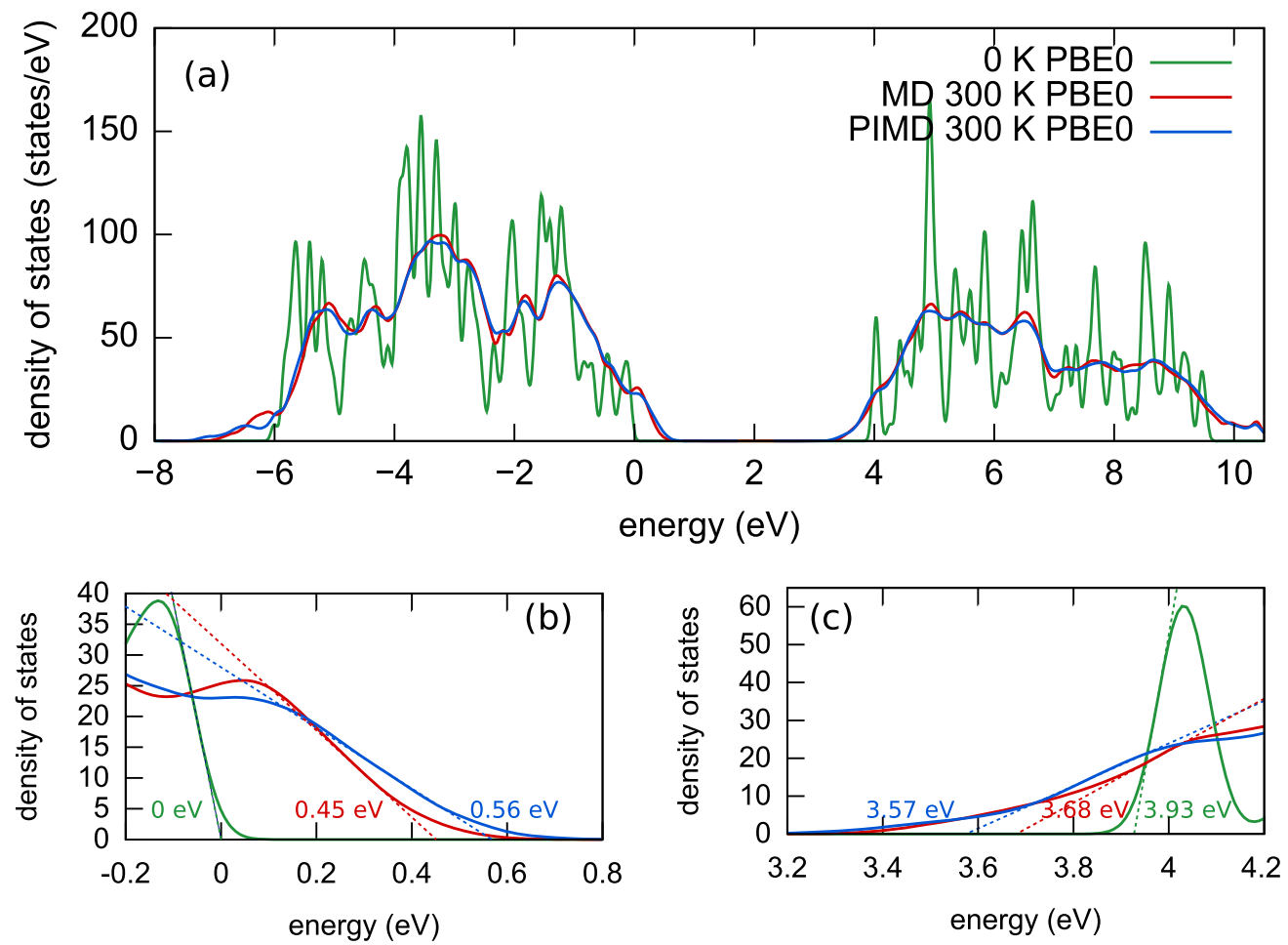

FIG. 3. (a) Comparison of the density of states (DOS) of $\mathrm{BiVO}_{4}$ calculated using PBE0 at $0 \mathrm{~K}$ and taking averages over the MD and PIMD trajectories at $300 \mathrm{~K}$. We use a broadening of $0.05 \mathrm{eV}$ to generate the plots. The DOS are aligned through the V $3 s$ level. The insets in (b) and (c) show the valence and conduction edges, respectively. Dashed lines indicate the linear extrapolations used to determine the positions of the VBM and CBM.

set to 0.48 fs. To overcome the effect of the band tail [39], we extract the band gap from the MD simulations through linear extrapolations of the wings of the electronic density of states (DOS) near the band edges. We verify if the DOS calculated at the $\Gamma$ point yields converged band edges. This is done by comparing the density of states calculated in the supercell and in a unit cell with $4 \times 4 \times 4$ and $6 \times 6 \times 6$ k-point meshes. We observe that the shape of the band edges is not affected by the use of a denser Brillouin zone sampling. From the linear extrapolation of the DOS, we obtain a closing of the band gap by $0.38 \mathrm{eV}$ from the MD with classical nuclei.

In the MD simulation with classical nuclei, the quantum zero-point (ZP) motion is neglected, while it can also lead to band gap renormalization [40-43]. To account for the nuclear quantum motions, we carry out path-integral molecular dynamics (PIMD), using the i-PI wrapper for the nuclear degrees of freedom [44] with interatomic forces taken from a semilocal DFT functional, as implemented in the $\mathrm{CP} 2 \mathrm{~K}$ package. To achieve the convergence of the PIMD results with only a few beads, we use a thermostat based on a generalized Langevin equation [45]. Calculations performed with four and six beads yield no significant differences. We present here the results obtained with six beads, averaged over a trajectory lasting 4 ps. The molecular dynamics simulations are preceded by an equilibration with classical nuclei. Taking into account the NQEs at $300 \mathrm{~K}$ leads to a total band gap closing of $0.57 \mathrm{eV}$. This renormalization is calculated using a semilocal functional. However, since this scheme yields a much lower band gap than the higher levels of theory, it could also underestimate the band gap renormalization. Therefore, we perform additional electronic structure calculations using the PBE0 functional on 720 snapshots chosen from the MD and PIMD trajectories. We obtain a band gap closing of $0.70 \mathrm{eV}$ with classic nuclei and of $0.92 \mathrm{eV}$ when including NQEs. In Fig. 3, we compare the respective densities of states to the result calculated at $0 \mathrm{~K}$.

Spin-orbit coupling, nuclear quantum effects, and finitetemperature vibrations lead to a total renormalization of the fundamental band gap by $0.70 \mathrm{eV}$ at the semilocal level and by $1.05 \mathrm{eV}$ at the PBE0 level. The corrected values of the fundamental band gap $E_{\mathrm{g}}^{\text {corr. }}$ of $\mathrm{BiVO}_{4}$ have been added to Table I. For both hybrid functional and various $G W$ methods we apply the renormalization calculated within PBE0. We note that after taking into account all these effects, the PBE functional underestimates the experimental band gap by about $1 \mathrm{eV}$. The band gap calculated with the hybrid functional HSE falls below the range of experimental data, while the hybrid functional PBE0 slightly overestimates the band gap. The various types of $G W$ methods predict band gaps in overall good agreement with the experimental values. We note that recently, the HSE functional with a fraction of Fock exchange reduced from $25 \%$ to $17 \%$ was used for high-throughput screening of vanadates for photoanode materials [2]. This reduction was based on the apparent success of such a scheme in reproducing the experimental band gap of a few metal vanadates, including $\mathrm{BiVO}_{4}$. From our findings it is clear that the agreement between HSE calculations with a reduced mixing parameter and experiment are due to fortuitous error cancellations, which cannot be expected to occur systematically across the whole class of metal vanadates. 
The band gap of a semiconductor detected through absorption spectroscopy corresponds to the optical gap. Up to now, we only focused on the fundamental band gap. To relate the two, we solve the Bethe-Salpeter equation (BSE) [46] within the $G W$ approximation, using the DP-CODE [47]. We include 12 valence and 20 conduction bands to obtain converged results. The independent-particle susceptibility, required as input, is constructed using the quasiparticle energies from the QSG $\tilde{W}$ calculation, including additional shifts coming from the spin-orbit coupling. We do not account for the indirect absorption contributions arising from phonon-assisted interband transitions. Such contributions can be included in ab initio calculations [48,49], but would not play a significant role in the absorption spectrum of $\mathrm{BiVO}_{4}$, since the dominating direct transition occurs at only $0.16 \mathrm{eV}$ higher energies than the indirect one. From the BSE calculations, we extract the exciton binding energy and the imaginary part of the macroscopic dielectric function $\varepsilon(\omega)$ of $\mathrm{BiVO}_{4}$. This dielectric function is calculated using the microscopic dielectric matrix $\varepsilon(\mathbf{q}, \omega)_{\mathbf{G}, \mathbf{G}^{\prime}}$,

$$
\varepsilon(\omega)=\lim _{\mathbf{q} \rightarrow 0} \frac{1}{\varepsilon(\mathbf{q}, \omega)_{\mathbf{G}=0, \mathbf{G}^{\prime}=0}^{-1}},
$$

where $\mathbf{q}$ lies within the first Brillouin zone and $\mathbf{G}, \mathbf{G}^{\prime}$ are reciprocal lattice vectors. To obtain converged results, a matrix dimension of $447 \times 447$ reciprocal lattice vectors $\mathbf{G}$ is required. In Fig. 4(a), we compare the imaginary part of the dielectric function calculated with and without excitonic effects. From the shifts of the eigenvalues, we find an exciton binding energy of $0.11 \mathrm{eV}$, an amount by which the optical band gap is reduced with respect to the fundamental one. This value is two times smaller than estimated by Kim et al. [11] using a hydrogen-like model. This is partially due to the fact that this model assumes parabolic electron and hole bands, which instead show a complicated dependence on the $k$ vector in reciprocal space (see Fig. 1).

To compare with spectroscopy experiments, we focus on the absorption coefficient $\alpha$. This is obtained by combining the imaginary part of the dielectric function $\left(\varepsilon_{\mathrm{im}}\right)$ resulting from the BSE calculation with the real part $\left(\varepsilon_{\mathrm{re}}\right)$ that is derived from the susceptibility used in the QS $G \tilde{W}$ calculation:

$$
\alpha(\omega)=\frac{4 \pi \omega}{c} \sqrt{\frac{\sqrt{\varepsilon_{\mathrm{re}}^{2}(\omega)+\varepsilon_{\mathrm{im}}^{2}(\omega)}-\varepsilon_{\mathrm{re}}(\omega)}{2}},
$$

where $c$ is the speed of light. In Fig. 4(b), we compare the calculated absorption coefficient with experimental data from Cooper et al. [10] and from Stoughton et al. [8]. We remark that in the absorption spectrum, we include the broadenings and shifts of the eigenvalues extracted from the PIMD at $300 \mathrm{~K}$. We apply an additional broadening of $0.14 \mathrm{eV}$, corresponding to the experimental resolution [8]. We find that the position of the first peak at $3 \mathrm{eV}$ in the calculated absorption spectrum is in very good agreement with its experimental counterpart. Additionally, the calculated intensity above the absorption edge falls well within the range of the experimental spectra. Below $2.5 \mathrm{eV}$, the agreement between the calculations and experiment is less impressive. However, discrepancies are already present between the two measured spectra. We suggest that these differences can be explained by the presence of defects or surface states in the measured samples.
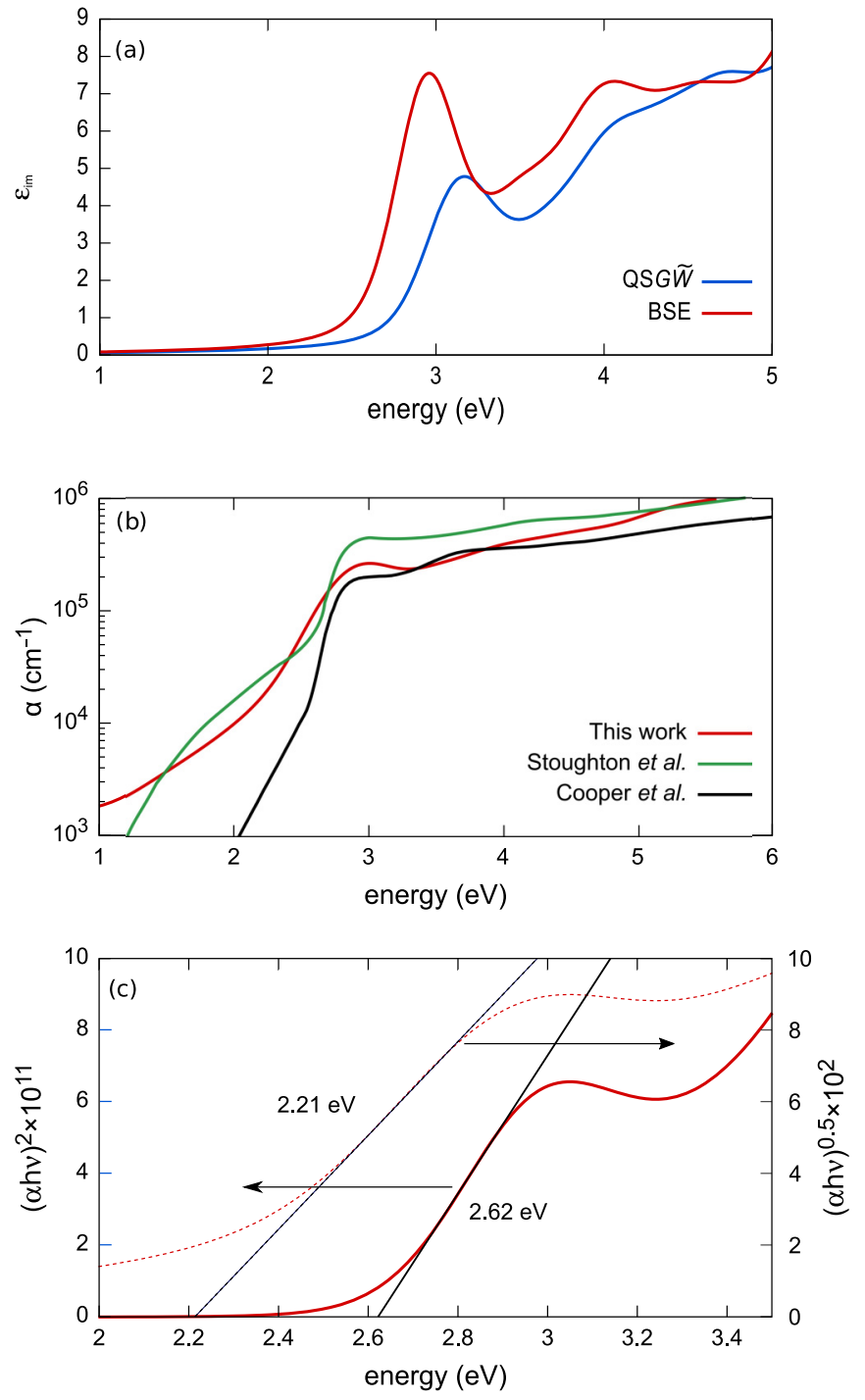

FIG. 4. (a) The imaginary part of the dielectric function calculated without excitonic effects $(\mathrm{QS} G \tilde{W})$ and with excitonic effects (BSE). (b) Comparison between calculated and measured optical absorption spectra. The experimental data are taken from Refs. [8] and [10]. (c) Tauc plots derived from the calculated absorption spectrum. The black lines indicate linear extrapolations of the absorption edges.

In experimental studies, the band gap of $\mathrm{BiVO}_{4}$ is extracted from UV-vis optical absorption spectra [8,10] through linear extrapolation of Tauc plots [25]. When the gap of a semiconductor can be described by a simple two-band model without excitonic effects, direct and indirect gaps can be extracted from the linearity of the plots of $(\alpha h \nu)^{2}$ and $(\alpha h v)^{0.5}$, respectively [50]. This approach was applied by Cooper et al. [10] to bismuth vanadate. However, our BSE calculations show that the excitonic effects are sizable in $\mathrm{BiVO}_{4}$. In this case, the absorption edge corresponding to the indirect transition has the same energy dependence as the direct transition without excitonic effects [50] and only the quadratic dependence can be used to extract the gap. This means that direct and indirect gaps cannot be distinguished using Tauc plots in $\mathrm{BiVO}_{4}$. 
We plot $(\alpha h v)^{2}$ in Fig. 4(c). From the linear extrapolation, we extract a band gap of $2.62 \mathrm{eV}$, in good agreement with $2.68 \mathrm{eV}$ obtained from the same type of plot by Cooper et al. [10]. We note that the value of the band gap extracted from the Tauc plot is slightly larger than the value of $2.48 \mathrm{eV}$ obtained from the difference of single-particle energy levels (see Table I). This means that band gaps obtained from Tauc plots for $\mathrm{BiVO}_{4}$ are slightly overestimated. Limitations associated with the use of Tauc plots have already been observed for other materials [51,52]. We also verify the effect of the experimental resolution in the absorption measurements on the band gap. This is done by not considering the experimental broadening of $0.14 \mathrm{eV}$ in the calculated spectrum. In this way, we find that the band gap only slightly increases from 2.62 to $2.66 \mathrm{eV}$. We note that using a Lorentzian broadening instead of a Gaussian one does not affect the extracted band gap.

In Fig. 4(c), we also illustrate the consequences of using the $(\alpha h v)^{0.5}$ plot for the band gap extraction. Even though we do not include indirect transitions in the calculations, we observe a linear region in the plot, leading to an extrapolated value of $2.21 \mathrm{eV}$. However, this value does not correspond to any energy transition in our calculations. This shows that the $(\alpha h v)^{0.5} \mathrm{de}-$ pendence can lead to a spurious gap in the case of $\mathrm{BiVO}_{4}$. Consequently, the value of $2.52 \mathrm{eV}$ inferred by Cooper et al. using the $(\alpha h v)^{0.5}$ dependence should not be assigned to an indirect gap.
In conclusion, we studied the electronic structure of $\mathrm{BiVO}_{4}$ at various levels of theory. We took into account a series of effects that affect the band gap, i.e., spin-orbit coupling, electron-hole interaction, nuclear quantum effects, and thermal vibrations. We showed that the apparent agreement with experiment achieved with semilocal functionals or hybrid functionals with a low fraction of the Fock exchange is due to error cancellation. We found that after including the corrections, the PBE functional strongly underestimates the band gap, while the higher levels of theory yield results in very good agreement with measured values. To address optical spectra, we considered excitonic effects and found them to be sizable. Their inclusion brings the calculated optical band gap and absorption spectrum in excellent agreement with experiment. Additionally, we investigated the use of Tauc plots. In particular, we pointed out that the use of the $(\alpha h v)^{0.5}$ plot leads to a spurious transition energy rather than to the indirect band gap.

The authors acknowledge the financial support from the Swiss National Science Foundation (SNSF) (Grants Nos. 200020-152799 and 200020-172524). This work has been realized in relation to the National Center of Competence in Research (NCCR) "Materials' Revolution: Computational Design and Discovery of Novel Materials (MARVEL)" of the SNSF. We used computational resources of the Swiss National Supercomputing Centre and of the Ecole Polytechnique Fédérale de Lausanne.
[1] L. Zhou, Q. Yan, A. Shinde, D. Guevarra, P. F. Newhouse, N. Becerra-Stasiewicz, S. M. Chatman, J. A. Haber, J. B. Neaton, and J. M. Gregoire, Adv. Energy Mater. 5, 1500968 (2015).

[2] Q. Yan, J. Yu, S. K. Suram, L. Zhou, A. Shinde, P. F. Newhouse, W. Chen, G. Li, K. A. Persson, J. M. Gregoire, and J. B. Neaton, Proc. Natl. Acad. Sci. USA 114, 3040 (2017).

[3] A. Kudo, K. Omori, and H. Kato, J. Am. Chem. Soc. 121, 11459 (1999).

[4] J. Yu and A. Kudo, Adv. Funct. Mater. 16, 2163 (2006).

[5] H. Luo, A. H. Mueller, T. M. McCleskey, A. K. Burrell, E. Bauer, and Q. Jia, J. Phys. Chem. C 112, 6099 (2008).

[6] Y. Park, K. J. McDonald, and K.-S. Choi, Chem. Soc. Rev. 42, 2321 (2013).

[7] D. Payne, M. Robinson, R. Egdell, A. Walsh, J. McNulty, K. Smith, and L. Piper, Appl. Phys. Lett. 98, 212110 (2011).

[8] S. Stoughton, M. Showak, Q. Mao, P. Koirala, D. Hillsberry, S. Sallis, L. Kourkoutis, K. Nguyen, L. Piper, D. Tenne, N. Podraza, D. Muller, C. Adamo, and D. Schlom, APL Mater. 1, 042112 (2013).

[9] J. K. Cooper, S. Gul, F. M. Toma, L. Chen, P.-A. Glans, J. Guo, J. W. Ager, J. Yano, and I. D. Sharp, Chem. Mater. 26, 5365 (2014).

[10] J. K. Cooper, S. Gul, F. M. Toma, L. Chen, Y.-S. Liu, J. Guo, J. W. Ager, J. Yano, and I. D. Sharp, J. Phys. Chem. C 119, 2969 (2015).

[11] T. W. Kim, Y. Ping, G. A. Galli, and K.-S. Choi, Nat. Commun. 6, 8769 (2015).

[12] M. Long, W. Cai, and H. Kisch, J. Phys. Chem. C 112, 548 (2008).
[13] K. Sayama, A. Nomura, Z.Zou, R. Abe, Y. Abe, and H. Arakawa, Chem. Commun. 2908 (2003).

[14] K. Sayama, A. Nomura, T. Arai, T. Sugita, R. Abe, M. Yanagida, T. Oi, Y. Iwasaki, Y. Abe, and H. Sugihara, J. Phys. Chem. B 110, 11352 (2006).

[15] A. Walsh, Y. Yan, M. N. Huda, M. M. Al-Jassim, and S.-H. Wei, Chem. Mater. 21, 547 (2009).

[16] Z. Zhao, Z. Li, and Z. Zou, Phys. Chem. Chem. Phys. 13, 4746 (2011).

[17] N. Wadnerkar and N. J. English, Comput. Mater. Sci. 74, 33 (2013).

[18] K. Ding, B. Chen, Y. Li, Y. Zhang, and Z. Chen, J. Mater. Chem. A 2, 8294 (2014).

[19] R. Crespo-Otero and A. Walsh, J. Phys. Chem. Lett. 6, 2379 (2015).

[20] J. P. Perdew, K. Burke, and M. Ernzerhof, Phys. Rev. Lett. 77, 3865 (1996).

[21] J. Heyd, J. E. Peralta, G. E. Scuseria, and R. L. Martin, J. Chem. Phys. 123, 174101 (2005).

[22] J. Heyd, G. E. Scuseria, and M. Ernzerhof, J. Chem. Phys. 118 8207 (2003).

[23] J. P. Perdew, M. Ernzerhof, and K. Burke, J. Chem. Phys. 105, 9982 (1996).

[24] L. Hedin, Phys. Rev. 139, A796 (1965).

[25] J. Tauc, R. Grigorovici, and A. Vancu, Phys. Status Solidi B 15, 627 (1966).

[26] J. Heyd, G. E. Scuseria, and M. Ernzerhof, J. Chem. Phys. 124, 219906 (2006).

[27] W. Chen and A. Pasquarello, Phys. Rev. B 92, 041115 (2015). 
[28] D. R. Hamann, Phys. Rev. B 88, 085117 (2013).

[29] A. Sleight, H.-Y. Chen, A. Ferretti, and D. Cox, Mater. Res. Bull. 14, 1571 (1979).

[30] S. Lebègue, B. Arnaud, M. Alouani, and P. E. Bloechl, Phys. Rev. B 67, 155208 (2003).

[31] M. Stankovski, G. Antonius, D. Waroquiers, A. Miglio, H. Dixit, K. Sankaran, M. Giantomassi, X. Gonze, M. Côté, and G.-M. Rignanese, Phys. Rev. B 84, 241201 (2011).

[32] X. Gonze, J.-M. Beuken, R. Caracas, F. Detraux, M. Fuchs, G.-M. Rignanese, L. Sindic, M. Verstraete, G. Zerah, F. Jollet, M. Torrent, A. Roy, M. Mikami, P. Ghosez, J.-Y. Raty, and D. Allan, Comput. Mater. Sci. 25, 478 (2002).

[33] X. Gonze, B. Amadon, P.-M. Anglade, J.-M. Beuken, F. Bottin, P. Boulanger, F. Bruneval, D. Caliste, R. Caracas, M. Côté, T. Deutsch, L. Genovese, P. Ghosez, M. Giantomassi, S. Goedecker, D. Hamann, P. Hermet, F. Jollet, G. Jomard, S. Leroux, M. Mancini, S. Mazevet, M. Oliveira, G. Onida, Y. Pouillon, T. Rangel, G.-M. Rignanese, D. Sangalli, R. Shaltaf, M. Torrent, M. Verstraete, G. Zerah, and J. Zwanziger, Comput. Phys. Commun. 180, 2582 (2009).

[34] X. Gonze, F. Jollet, F. A. Araujo, D. Adams, B. Amadon, T. Applencourt, C. Audouze, J.-M. Beuken, J. Bieder, A. Bokhanchuk, E. Bousquet, F. Bruneval, D. Caliste, M. Ct, F. Dahm, F. D. Pieve, M. Delaveau, M. D. Gennaro, B. Dorado, C. Espejo, G. Geneste, L. Genovese, A. Gerossier, M. Giantomassi, Y. Gillet, D. Hamann, L. He, G. Jomard, J. L. Janssen, S. L. Roux, A. Levitt, A. Lherbier, F. Liu, I. Lukaevi, A. Martin, C. Martins, M. Oliveira, S. Ponc, Y. Pouillon, T. Rangel, G.-M. Rignanese, A. Romero, B. Rousseau, O. Rubel, A. Shukri, M. Stankovski, M. Torrent, M. V. Setten, B. V. Troeye, M. Verstraete, D. Waroquiers, J. Wiktor, B. Xu, A. Zhou, and J. Zwanziger, Comput. Phys. Commun. 205, 106 (2016).

[35] A. Riefer, M. Friedrich, S. Sanna, U. Gerstmann, A. Schindlmayr, and W. G. Schmidt, Phys. Rev. B 93, 075205 (2016).
[36] J. VandeVondele, M. Krack, F. Mohamed, M. Parrinello, T. Chassaing, and J. Hutter, Comput. Phys. Commun. 167, 103 (2005).

[37] J. VandeVondele and J. Hutter, J. Chem. Phys. 127, 114105 (2007).

[38] S. Goedecker, M. Teter, and J. Hutter, Phys. Rev. B 54, 1703 (1996).

[39] F. Ambrosio, G. Miceli, and A. Pasquarello, J. Chem. Phys. 143, 244508 (2015).

[40] M. Cardona and M. Thewalt, Rev. Mod. Phys. 77, 1173 (2005).

[41] G. Antonius, S. Poncé, P. Boulanger, M. Côté, and X. Gonze, Phys. Rev. Lett. 112, 215501 (2014).

[42] G. Antonius, S. Poncé, E. Lantagne-Hurtubise, G. Auclair, X. Gonze, and M. Côté, Phys. Rev. B 92, 085137 (2015).

[43] M. Friedrich, A. Riefer, S. Sanna, W. Schmidt, and A. Schindlmayr, J. Phys.: Condens. Matter 27, 385402 (2015).

[44] M. Ceriotti, J. More, and D. E. Manolopoulos, Comput. Phys. Commun. 185, 1019 (2014).

[45] M. Ceriotti, G. Bussi, and M. Parrinello, Phys. Rev. Lett. 103, 030603 (2009).

[46] R. E. Cutkosky, Phys. Rev. 96, 1135 (1954).

[47] V. Olevano, L. Reining, and F. Sottile, DP-Code, http://www.dpcode.org/.

[48] J. Noffsinger, E. Kioupakis, C. G. Van de Walle, S. G. Louie, and M. L. Cohen, Phys. Rev. Lett. 108, 167402 (2012).

[49] M. Zacharias and F. Giustino, Phys. Rev. B 94, 075125 (2016).

[50] P. Yu and M. Cardona, Fundamentals of Semiconductors: Physics and Materials Properties, 4th ed. (Springer, Heidelberg, 2010).

[51] M. Meinert and G. Reiss, J. Phys.: Condens. Matter 26, 115503 (2014).

[52] H. Jiang, J. Phys. Chem. C 116, 7664 (2012). 